\title{
Effect of Intermittent Pneumatic Compression in Combination with Kinesiotape on Post Mastectomy Lymphedema
}

\author{
Hayam A. Diab*1, Haidy N. Asham¹, Nancy H. Aboelnour ${ }^{1}$, Hagar A. Alagizy ${ }^{2}$ \\ ${ }^{1}$ Department of Physical Therapy for Surgery, Faculty of Physical Therapy, Cairo University, Egypt. \\ ${ }^{2}$ Department of Oncology and Nuclear Medicine, Faculty of Medicine, Menoufia University, Egypt. \\ *Corresponding author: Hayam A. Diab, Mobile: (+20) 01092710752, E-Mail: haiamdiab519@ gmail.com
}

\begin{abstract}
Background: Lymphedema caused by breast cancer is one of the side effects of therapy. It is defined as arm edema in breast cancer patients caused by surgery or radiation therapy interrupting the flow of the axillary lymphatic system, resulting in fluid accumulation in the subcutaneous tissue of the arm, decreased tissue distensibility around the joints, and increased weight of the extremity.

Objective: This study was conducted to detect the effect of combination of intermittent pneumatic compression and kinesiotape on post mastectomy lymphedema.

Patients and methods: Thirty females diagnosed with post mastectomy lymphedema were assigned randomly into two equal groups. Group A (Study group) received intermittent pneumatic compression (IPC) with kinesiotape (KT) in addition to complete decongestive therapy (manual lymphatic drainage-compression bandage-medical exercises-skin care) three times per week for 4 weeks. While, Group B (Control group) received only complete decongestive therapy 3 times per week for 4 weeks. Assessment of lymphedema and shoulder range of motion (flexion-abduction-external rotation) were done by tape measurement and goniometer respectively before and after 4 weeks.

Results: There was no significant difference in lymphedema size and shoulder range of motion (ROM) between groups pre-treatment $(\mathrm{p}>0.05)$. Comparison between groups post treatment revealed a significant decrease in lymphedema size and a significant increase in shoulder ROM of the study group compared with that of the control group (p <0.05). Conclusion: The results suggested that IPC in combination with KT was an effective method in post mastectomy lymphedema more than complete decongestive therapy only.
\end{abstract}

Keywords: Intermittent pneumatic compression, Kinesiotape, Post mastectomy lymphedema.

\section{INTRODUCTION}

Mastectomy is the surgical removal of the cancerous portion of the breast tissue (malignant tumor) as well as a margin of normal tissue surrounding it before normal margins are found (i.e., no cancer cells are found at the edges of the tissue removed ${ }^{(\mathbf{1})}$.

Modified radical mastectomy is one of the main methods of clinical treatment for breast cancer, in which the purpose to thoroughly eliminating breast cancer is achieved by removing the lesions and adjacent tissues of breast cancer. Modified radical mastectomy can be classified into 2 types: type 1 is Patey's operation, in which the pectoralis major is preserved; another type is Auchincloss's operation, in which the pectoralis major and pectoralis minor are retained. In the latter, the axillary lymph nodes can also be thoroughly dissected while preserving the pectoralis major and pectoralis minor ${ }^{(2)}$. At present, modified radical mastectomy is usually applied to patients with stage I and II breast cancer, especially in patients with lesions distributed in the lateral breast and patients with axillary lymph node metastases, However, in modified radical mastectomy, all breast tissues must be removed ${ }^{(3)}$.

Breast cancer related lymphedema (BCRL) is one of the most commonly reported complications of treatment for the estimated 2 million breast cancer cases worldwide annually with an incidence of $17 \%{ }^{(4)}$. Lymphedema is the accumulation of protein-rich fluid in the affected body part, which induces constant swelling and obstructs the flow of fluid ${ }^{(5)}$. The most well-known causes of lymphedema are radiotherapy and lymph node dissection in patients with breast cancer. Large axillary disease, obesity, and positive axillary lymph nodes are also risk factors for lymphedema ${ }^{(6)}$. Upper limb lymphedema is the most common cause of morbidity after mastectomies with axillary lymph node removal. This condition is characterized by the abnormal accumulation of fluids and proteins in the intercellular space, chronic inflammation, and edema ${ }^{(7)}$. Lymphedema is classified as either primary or secondary. Primary lymphedema is caused by error in lymphatic growth and is idiopathic. Secondary lymphedema develops after an injury to the lymphatic system, which is usually established ${ }^{(8)}$.

Intermittent pneumatic compression (IPC) is a noninvasive procedure that has been shown to be beneficial in clinical practice. IPC is also commonly used in the treatment of extremity edema as well as venous thromboembolism prevention in patients with a high risk of bleeding and venous ulcers ${ }^{(9)}$. IPC is a multi-chambers, sequential inflation, gradient inflation pressure, time of inflation sufficient for translocation of tissue fluid to proximal region, no deflation of distal chambers to prevent fluid back-flow and venous stasis in the superficial limb system (10). Pneumatic compression therapy has been used extensively to aid in 
the management of lymphedema. It applies pressure to the extremity, causing edema to migrate into the limb's root and into the adjacent trunk quadrant due to a pressure gradient ${ }^{(\mathbf{1 1})}$.

Kinesiotape (KT) is an elastic cotton strip with an acrylic adhesive that is used to relieve the discomfort and disability associated with sports injuries and a number of other physical conditions ${ }^{(12)}$. Kinesiotape aids in edema reduction and lymphatic drainage improvement. It draws the upper layers of the skin away from the muscles, allowing space between the dermis and the muscles, relieving pressure on the lymphatic and blood vessels and improving lymphatic drainage (13).

Lymphedema post mastectomy remains a challenging problem, as it causes feelings of heaviness, swelling, decreased flexibility or tightness in the hand, arm, chest, breast, or underarm areas and psychological problems. Early treatment of lymphedema is important (14). Therefore, this study was developed to examine the effect of intermittent compression in combination with kinesiotape for reducing post mastectomy lymphedema as a conservative treatment modalities.

\section{PATIENTS AND METHODS}

Thirty patients diagnosed with post mastectomy lymphedema were referred to outpatient clinic from Clinical Oncology and Nuclear Medicine Department, Faculty of Medicine, Menoufia University, and participated in this study. Diagnosis of lymphedema was done by referring oncologist.

Patients were enrolled in this trial if they met the following criteria: (1) Age >18 years. (2) All patients suffered from unilateral stage 2 lymphedema. (3) All patients had more than $2 \mathrm{~cm}$ difference between two limbs at circumference measurement. (4) All patients had completed chemotherapy and radiotherapy.

The exclusion criteria included: (1) Skin infection (2) Current limb ischemia, venous thrombosis (3) Systemic edema due to impaired heart, kidney and liver function

(4) Current metastases.

Ethical consideration:

An approval of the study was obtained from Cairo University academic and ethical committee. Every patient signed an informed written consent for acceptance of the operation. This work has been carried out in accordance with The Code of Ethics of the World Medical Association (Declaration of Helsinki) for studies involving humans.

The patients were divided randomly into two equal groups. Randomization steps were conducted via the envelope mode. After patient agreement to participate in the study, cards with either 'IPC and KT' or 'CDT' recorded on them were put in envelopes. Envelops were closed; then a blinded physical therapist was asked to select one envelope. According to the selected card, patients were assigned to their corresponding group. Dates for starting the allocated therapy were regulated and the therapy was begun through the first week of randomization.

\section{Tape measurement:}

Tape (teufelGm bH/ Germany) was used for assessing circumference of limbs. Measurements were taken on both arms at five anatomic locations: (1) The axilla, (2) Halfway from the antecubital fossa to the axilla, (3) Antecubital fossa, (4) Halfway from the antecubital fossa to the wrist, and (5) The wrist.

\section{Goniometer:}

Goniometer (GONIOMETER/India) was used to measure shoulder range of motion (flexion-abductionexternal rotation). For measurement; the patient was placed supine with the thorax firmly strapped to the table to prevent body shift, which would tend to compensate for movement of the shoulder.

\section{For shoulder flexion:}

- Goniometer alignment: Axis - center of humeral head near acromion process. Stationary arm parallel mid-axillary line.

Moving arm - aligned with midline of humerus (lateral epicondyle).

\section{For shoulder abduction:}

- Goniometer alignment: Axis - center of humeral head near acromion process. Stationary arm parallel to trunk

Moving arm - aligned with midline of humerus

For shoulder external rotation:

- Goniometric alignment: axis-olecranon process.

Stationary arm- parallel to the floor

Moving arm- parallel with the forearm

\section{Treatment:}

Both groups received complete decongestive therapy (manual lymphatic drainage-bandages-exercises-skin care) 3 times per week for 4 weeks. The experimental group additionally received: (1) IPC (power-Q 1000 model| WHF. 324| China) with the following parameters; Pressure: $60 \mathrm{mmHg}, 30 \mathrm{~min}, 3$ times per week for 4 weeks. (2) KT (Ares/Japan) the tape was applied only to the upper extremity in a double fan shape within the arm and forearm. It was maintained for 24 hours/day during four weeks and replaced every seven days. All patients were evaluated before the start of the treatment and after 4 weeks.

\section{Statistical analysis}

All statistical analysis was conducted through the statistical package for the social sciences (SPSS) version 25 for windows (IBM SPSS, Chicago, IL, USA). Quantitative data were represented as means \pm standard deviation (SD). Normal distribution of data was checked using the Shapiro-Wilk test for all variables. Levene's test for homogeneity of variances was conducted to test the homogeneity between groups. Unpaired t-test was conducted to compare the two groups. Paired t-test was conducted for comparison 
between pre and post treatment in each group. The level of significance for all statistical tests was set at $\mathrm{p}<0.05$.

\section{RESULTS}

Subject characteristics: Thirty patients with post mastectomy lymphedema participated in this study. Most of them were done modified radical mastectomy. The mean \pm SD age of study group was $41.2 \pm 6.62$ years, with maximum value of 54 years and minimum value of 35 years. The mean \pm SD age of control group was $40.66 \pm 7.17$ years, with maximum value of 54 years and minimum value of 31 years. There was no significant difference between both groups in the mean age values $(\mathrm{p}=0.83)$.

Effect of treatment on lymphedema size and shoulder ROM:

\section{Within group comparison:}

There was a significant decrease in lymphedema size post treatment compared with that pretreatment in the study and control groups $(\mathrm{p}<0.001)$.
The percent of decrease in lymphedema size at axilla, mid way between cubital Fossa and axilla, cubital fossa, mid way between cubital fossa and wrist and wrist in study group was 16.97, 17.85, 14.17, 19.28 and $16.06 \%$ respectively, while that in control group was 7.01, 8.07, 7.6, 9.98 and 7.58 respectively (Table 1 and Figure 1).

There was a significant increase in shoulder ROM post treatment compared with that pretreatment in the study and control groups $(p<0.001)$. The percent of increase in shoulder abduction, flexion and external rotation of study group was $6.84,7.11$ and $10.73 \%$ respectively, while that in control group was 4.09, 4.31 and $9.46 \%$ respectively (Table 2 and Figure 2). There was no significant difference in lymphedema size and shoulder ROM between groups pre-treatment.

Between groups comparison: Comparison between groups post treatment revealed a significant decrease in lymphedema size and a significant increase in shoulder ROM of the study group compared with that of the control group (Tables 1, 2 and Figures 1, 2).

Table (1): Mean lymphedema size pre and post treatment of the study and control groups.

\begin{tabular}{|c|c|c|c|}
\hline Lymphedema size (cm) & $\begin{array}{c}\begin{array}{c}\text { Study group } \\
\mathrm{N}=15\end{array} \\
\text { mean } \pm \mathrm{SD}\end{array}$ & $\begin{array}{c}\begin{array}{c}\text { Control group } \\
\mathrm{N}=15\end{array} \\
\text { mean } \pm \mathrm{SD}\end{array}$ & p value \\
\hline Axilla & & & \\
\hline Pre treatment & $46.8 \pm 4.84$ & $45.66 \pm 5.7$ & 0.56 \\
\hline Post treatment & $38.86 \pm 3.24$ & $42.46 \pm 4.56$ & 0.02 \\
\hline \multicolumn{4}{|c|}{$\begin{array}{l}\text { Mid way between cubital } \\
\text { Fossa and axilla }\end{array}$} \\
\hline Pre treatment & $35.13 \pm 4.58$ & $35.46 \pm 4.27$ & 0.83 \\
\hline Post treatment & $28.86 \pm 3.18$ & $32.6 \pm 4.3$ & 0.01 \\
\hline \multicolumn{4}{|l|}{ Cubital fossa } \\
\hline Pre treatment & $30.13 \pm 2$ & $30.8 \pm 2.83$ & 0.46 \\
\hline Post treatment & $25.86 \pm 1.92$ & $28.46 \pm 3.37$ & 0.02 \\
\hline \multicolumn{4}{|c|}{$\begin{array}{l}\text { Mid way between cubital } \\
\text { fossa and wrist }\end{array}$} \\
\hline Pre treatment & $27.33 \pm 1.67$ & $26.66 \pm 2.58$ & 0.41 \\
\hline Post treatment & $22.06 \pm 1.83$ & $24 \pm 2.59$ & 0.03 \\
\hline \multicolumn{4}{|l|}{ Wrist } \\
\hline Pre treatment & $19.93 \pm 1.57$ & $19.4 \pm 1.68$ & 0.38 \\
\hline Post treatment & $16.73 \pm 1.43$ & $17.93 \pm 1.38$ & 0.03 \\
\hline
\end{tabular}

SD: Standard deviation 
Table (2): Mean shoulder ROM pre and post treatment of the study and control groups

\begin{tabular}{|c|c|c|c|}
\hline \multirow{3}{*}{$\begin{array}{l}\text { ROM (degrees) } \\
\text { Abduction }\end{array}$} & Study group (N=15) & Control group $(\mathrm{N}=15)$ & \multirow[b]{2}{*}{ p value } \\
\hline & mean \pm SD & mean \pm SD & \\
\hline & & & \\
\hline Pre treatment & $157.86 \pm 3.66$ & $158.06 \pm 3.05$ & 0.87 \\
\hline $\begin{array}{l}\text { Post treatment } \\
\text { Flexion }\end{array}$ & \multicolumn{3}{|c|}{ Flexion } \\
\hline Pre treatment & $155.6 \pm 3.71$ & $156.13 \pm 4.03$ & 0.71 \\
\hline Post treatment & $166.67 \pm 4.06$ & $162.86 \pm 3.41$ & 0.01 \\
\hline \multicolumn{4}{|l|}{ External rotation } \\
\hline Pre treatment & $75.86 \pm 3.06$ & $74.6 \pm 3.48$ & 0.29 \\
\hline Post treatment & $84 \pm 2.6$ & $81.66 \pm 2.31$ & 0.01 \\
\hline
\end{tabular}

SD: Standard deviation

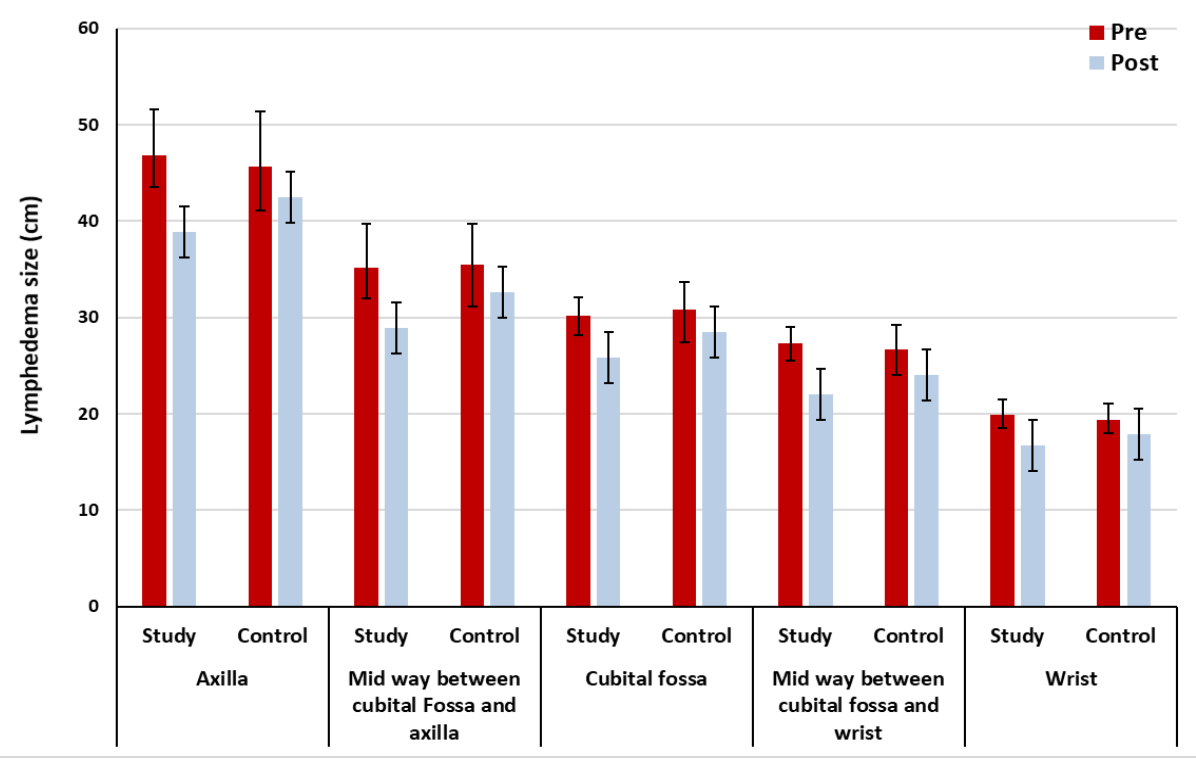

Figure (1): Mean lymphedema size pre and post treatment of the study and control groups

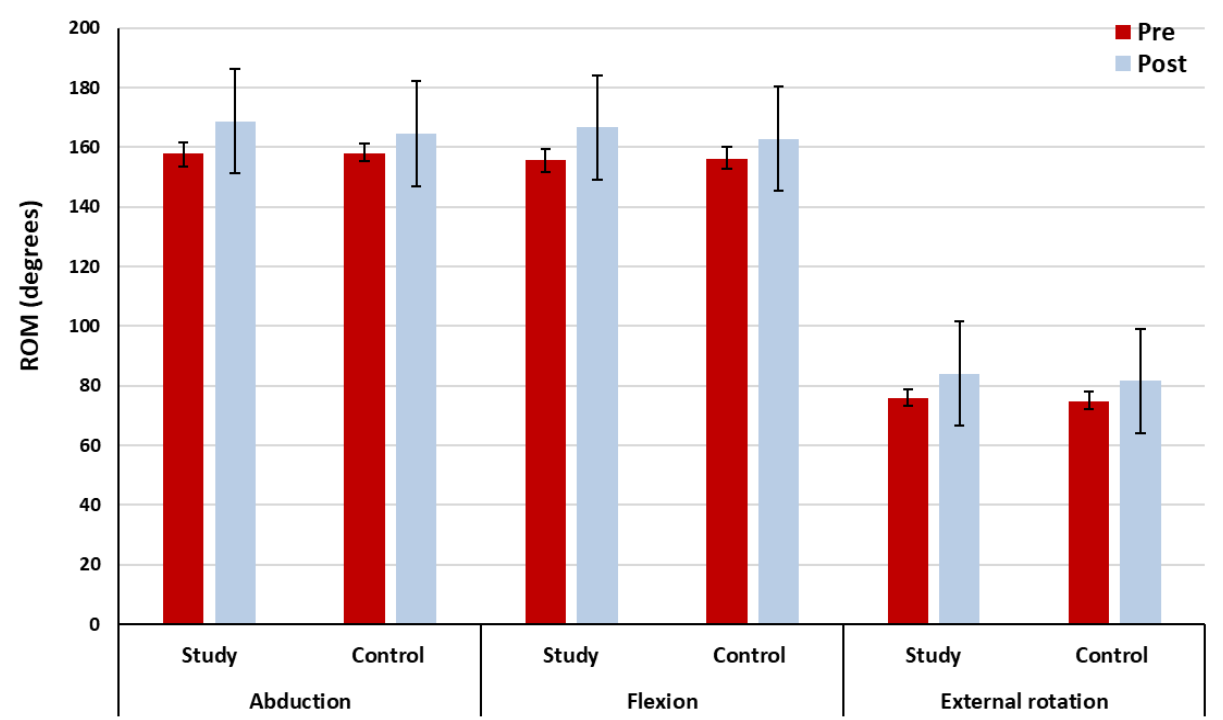

Figure (2): Mean shoulder ROM pre and post treatment of the study and control groups 


\section{DISCUSSION}

Intermittent pneumatic compression in combination with kinesiotape is one of the effective methods for management of lymph edema. In this study, there was a significant decrease in lymphedema size post treatment. The percent of decrease in lymphedema size at axilla, mid way between cubital Fossa and axilla, cubital fossa, mid way between cubital fossa and wrist and wrist in study group was 16.97, 17.85, 14.17, 19.28 and $16.06 \%$ respectively. There was a significant increase in shoulder ROM post treatment. The percent of increase in shoulder abduction, flexion and external rotation of study group was $6.84,7.11$ and $10.73 \%$ respectively. These outcomes may be due to pumping action of intermittent pneumatic compression, which facilitates the flow of lymph in lymphedema during compression ${ }^{(15)}$ and also the effect of kinesiotape as it enables the upper layers of the skin to be pulled, allowing space between the dermis and the muscle. Through this action, pressure exerted on the lymphatic vessels and channels is relieved, enhancing lymphatic flow ${ }^{(16)}$.

Intermittent pneumatic compression has been evaluated in several different studies as Nossair $\boldsymbol{e t}$ al. (17) who compared between compression therapy (group A) and laser therapy (group B). Assessment by tape measurements showed that improvement of arm circumference in group (A) was $31.34 \%$ while improvement of arm circumference in group (B) was $13.03 \%$. Also Kostanoglu et al. ${ }^{(18)}$ compared between manual lymphatic drainage with compression bandage (Group I) and IPC (Group II). Assessment by tape measurements showed that significant decrease in the total arm volume (12.2\% decrease in Group I and 14.9\% decrease in Group II).

Furthermore, Uzkeser et al. ${ }^{(19)}$ compared between complex decongestive physical therapy (CDT) (group 1) and CDT combined with IPC (group 2), patients were assessed according to circumference measurements of landmarks. Both treatments showed significant difference in both groups when comparing them before and after therapy. The baseline median volume difference of group 1 was 630, after therapy it was 480 . In group 2 , the beginning median volume difference was 840, and after therapy it was 500 . Szuba et al. (20) compared between decongestive lymphatic therapy only (DLT) and DLT with IPC. Patients were assessed by water displacement, tissue tonometry to assess elasticity of the skin, and goniometry to measure joint mobility. It was found that addition of IPC to standard DLT yielded an additional mean volume reduction $(45.3 \%$ vs. $26 \% ; P<0.05)$

Also kinesiotape has been evaluated in several different studies as Do et al. ${ }^{(21)}$ who studied the effectiveness of kinesiotaping, along with manual lymphatic drainage (MLD), exercise, and skin care to treat a patient with unilateral breast-cancer-related arm lymphedema and found that the excess edema volume decreased to $608 \mathrm{ml}$, meaning that the edema volume was reduced $79.5 \%$. Moreover, Tantawy et al. ${ }^{(22)}$ compared effect of kinesiotaping versus pressure garment (PG) in young females with post mastectomy lymphedema. The results showed that the limb circumferences improved after treatment in the KT group $(\mathrm{P}<0.05)$, while the $\mathrm{PG}$ group showed no significant improvement $\mathrm{p}>0.05$.

Our finding agreed with Pajero et al. ${ }^{(23)}$ who compared between kinesiotape and compression garment for breast cancer-related lymphedema and found that the decrease in the relative volume change was greater in the kinesiotaping intervention $(-5.7 \%$, $\mathrm{SD}=2.0)$ compared to that observed using compression garments $(-3.4 \%, \mathrm{SD}=2.9)(P<0.001)$. The range of motion of five upper-limb movements increased after applying taping (between $5.8^{\circ}$ and $\left.16.7^{\circ}\right)(P<0.05)$, but not after compression $(P>0.05)$.

From the previous discussion of these results and according to reports of researches in the field related to present study, it could be concluded that the results of this study support the expectation that the combination between IPC and KT play important role in lymphedema management and higher statistical results were reported in lymphedema size and shoulder range of motion than previous studies. The possible explanation for these results is combination of both IPC and KT modalities.

These outcomes may be due to: (1) Pneumatic compression allow translocation of retained fluid from the interstitial into the lymphatic lumen by producing a pressure gradient. (2) Pneumatic compression produced unidirectional flow without backflow. (3) It acts as "a muscle pump" which facilitate the flow of lymph in lymphedema. (4) KT enables the upper layers of the skin to be pulled, allowing space between the dermis and the muscle, through this action, pressure exerted on the lymphatic vessels and channels is relieved, enhancing lymphatic flow.

This study was restricted by small sample size, further researches are recommended to include larger sample size and advanced stages of lymphoedema to provide better statistical analysis evaluate and assure the effect of combination between IPC and KTto provide better statistical analysis evaluate and assure the effect of combination between IPC and KT. Lack of previous studies on combination between IPC and KT is another limitation, so more studies are recommended to investigate the effect of combination between IPC and KT on post mastectomy lymphedema. Psychological status of the patient was another limitation of this study.

\section{CONCLUSION}

Intermittent pneumatic compression in combination with kinesiotape is a safe method and had more significant effect on lymphedema reduction and 
improvement of shoulder range of motion than CDT alone.

\section{REFERENCES}

1. National Cancer Institute (2012): Dictionary of cancer terms. Retrieved June 4, 2012, from http://www.cancer.gov/dictionary

2. Lazaraviciute G, Chaturvedi S (2017): Mastectomy-A critical review. Open Journal of Clinical Diagnostics, 7(2): 58-66.

3. Freitas-Júnior $\mathbf{R}$, Oliveira $\mathrm{E}$, Pereira $\mathbf{R}$ et al. (2006): Modified radical mastectomy sparing one or both pectoral muscles in the treatment of breast cancer: intra and postoperative complications. Sao Paulo Medical Journal, 124, 130-134.

4. Franceschini G, Sanchez A, Di Leone A et al. (2015): Update on the surgical management of breast cancer. Ann Ital Chir., 86(2): 89-99.

5. DiSipio T, Rye S, Newman B et al. (2013): Incidence of unilateral arm lymphoedema after breast cancer: a systematic review and meta-analysis. The Lancet Oncology, 14(6):500-15.

6. Pyszora A, Krajnik M (2010): Is Kinesio taping useful for advanced cancer lymphedema treatment?' A case report. Adv Palliat Med., 9:141-144.

7. Gallagher S, O'Connor L, Roberts D et al. (2021): Common conservative treatments for lymphoedema post breast cancer: A focus article. Physiotherapy Practice and Research, 42(1): 7-11.

8. Fernandes A, Vidal G, Moreira C et al. (2013): Lymphedema in the mastectomy postoperative period: an integrative literature review. Adv Breast Cancer Res., 2(4):60-154.

9. Greene A, Goss J (2018): Lymphedema management: Diagnosis and staging of lymphedema. In Seminars in Plastic Surgery, 32(1): 12-16.

10. Onuigbo M (2010): Bilateral lower extremity sequential compression devices (SCDs): A novel approach to the management of intradialytic hypotension in the out-patient setting-Report of a case series. Ren Fail., 32:32-35.

11. Olszewski W, Jain P, Ambujam G et al. (2011): Tissue fluid pressure and flow during pneumatic. Physiothery J., 9: 58-89.

12. Feldman J, Stout N, Wanchai A et al. (2012): Intermittent pneumatic compression therapy: a systematic review. Lymphology, 45(1): 13-25.

13. Coopee $\mathbf{R}$ (2011): Elastic taping (Kinesio Taping Method) Rehabilitation of the hand and upper extremity, $6^{\text {th }}$ edn. Mosby, Philadelphia, Pp. 15391528.

14. Kasawara K, Mapa J, Ferreira V et al. (2018): Effects of Kinesio Taping on breast cancer-related lymphedema: A meta-analysis in clinical trials. Physiotherapy Theory and Practice, 34(5): 337345.

15. Ahmed R, Thomas W, Yee D et al. (2006): Randomized controlled trial of weight training and lymphedema in breast cancer survivors. J Clin Oncol., 24(18): 2765-72.

16. Yang J, Wu S, Chiang M et al. (2018): Intraoperative identification and definition of "functional" lymphatic collecting vessels for supermicrosurgical lymphaticovenous anastomosis in treating lymphedema patients. Journal of Surgical Oncology, 117(5): 9941000.

17. Pop T, Karczmarek-Borowska B, Tymczak M et al. (2014): The influence of Kinesiology Taping on the reduction of lymphoedema among women after mastectomy preliminary study. Contemporary Oncology, 18(2): 124-129.

18. Nossair A, Shahata S, Wahba E et al. (2018): Lowlevel laser versus pneumatic compression pump on lymphedema post mastectomy. Current Science Internationa, 7(4):481-485.

19. Kostanoglu G, Ozbas C, Cabioglu N et al. (2012): Comparison of intermittent pneumatic compression with manual lymphatic drainage for treatment of breast cancer-related lymphedema. Lymphatic Research and Biology, 10(3): 129-135.

20. Uzkeser H, Karatay S, Erdemci B et al. (2015): Efficacy of manual lymphatic drainage and intermittent pneumatic compression pump use in the treatment of lymphedema after mastectomy: a randomized controlled trial. Breast Cancer, 22(3): 300-307.

21. Szuba A, Achalu R, Rockson S (2002): Decongestive lymphatic therapy for patients with breast carcinoma-associated lymphedema: A randomized, prospective study of a role for adjunctive intermittent pneumatic compression. Cancer: Interdisciplinary International Journal of the American Cancer Society, 95(11): 2260-2267.

22. Do J, Jeon, J, Kim W (2017): The effects of bandaging with an additional pad and taping on secondary arm lymphedema in a patient after mastectomy. Journal of physical therapy science, 29(7): 1272-1275.

23. Tantawy S, Abdelbasset W, Nambi G et al. (2019): Comparative study between the effects of kinesio taping and pressure garment on secondary upper extremity lymphedema and quality of life following mastectomy: a randomized controlled trial. Integrative Cancer Therapies, 18(4): 1-10.

24. Pajero Otero V, Garcia Delgado E, Martin Cortijo C et al. (2019): Kinesio taping versus compression garments for treating breast cancer-related lymphedema: a randomized, cross-over, controlled trial. Clinical Rehabilitation, 33(12): 1887-1897. 